\title{
Positive Solutions Using Bifurcation Techniques for Boundary Value Problems of Fractional Differential Equations
}

\author{
Yansheng Liu \\ School of Mathematical Sciences, Shandong Normal University, Jinan 250014, China \\ Correspondence should be addressed to Yansheng Liu; yanshliu@gmail.com
}

Received 3 April 2013; Accepted 30 September 2013

Academic Editor: Soheil Salahshour

Copyright (C) 2013 Yansheng Liu. This is an open access article distributed under the Creative Commons Attribution License, which permits unrestricted use, distribution, and reproduction in any medium, provided the original work is properly cited.

\begin{abstract}
This paper is concerned with the existence of positive solutions for a class of boundary value problems of fractional differential equations with parameter. The main tools used here are bifurcation techniques and topological degree theory. Finally, an example is worked out to demonstrate the main result.
\end{abstract}

\section{Introduction}

During the last few decades, fractional calculus and fractional differential equations have been studied extensively since fractional-order models are found to be more adequate than integer-order models in some real-world problems. In fact, fractional derivatives provide an excellent tool for the description of memory and hereditary properties of various materials and processes. The mathematical modeling of systems and processes in the fields of physics, chemistry, aerodynamics, electrodynamics of complex medium, polymer rheology, and so forth involves derivatives of fractional order. For details and examples, see [1-7] and references therein. Recently, there have been a few papers which deal with the boundary value problem for fractional differential equation. For example, in [8], Tian and Liu investigated the following singular fractional boundary value problem (BVP, for short) of the form

$$
\begin{gathered}
{ }^{C} D_{0^{+}}^{\alpha} u(t)+\lambda f(t, u(t))=0, \quad 0<t<1, \\
u^{(j)}(0)=0, \quad 0 \leq j \leq n-1, \quad j \neq 2, \\
u^{\prime \prime}(1)=0,
\end{gathered}
$$

where ${ }^{C} D_{0^{+}}^{\alpha}$ is Caputo's fractional derivatives, $n-1<\alpha \leq n$, $n \geq 4$, and $f:(0,1) \times(0,+\infty) \rightarrow[0,+\infty)$ is continuous; that is, $f(t, u)$ may be singular at $t=0,1$ and $u=0$. By constructing a special cone, they obtained that there exist positive numbers $\lambda^{*}$ and $\lambda^{* *}$ with $\lambda^{*}<\lambda^{* *}$ such that the above system has at least two positive solutions for $\lambda \in\left(0, \lambda^{*}\right)$ and no solution for $\lambda>\lambda^{* *}$ under some suitable assumptions such as the following.

(A1) There exists an interval $[a, b] \subset(0,1)$ such that $\lim _{u \rightarrow+\infty} f(s, u) / u=+\infty$ uniformly with respect to $s \in[a, b]$.

In [9], Bai and Lü consider the following nonlinear fractional differential equation Dirichlet-type boundary value problem:

$$
\begin{gathered}
D_{0^{+}}^{\alpha} u(t)+f(t, u(t))=0, \quad t \in(0,1), \\
u(0)=u(1)=0,
\end{gathered}
$$

where $1<\alpha \leq 2$ is a real number and $D_{0^{+}}^{\alpha}$ is the standard Riemann-Liouville differentiation. The corresponding Green function is derived. By means of some fixed point theorems on cone, the existence and multiplicity of positive solutions for BVP (2) were investigated.

In [10], Jiang and Yuan further investigated BVP (2). Comparing with [9], they deduced some new properties of the Green function, which extended the results of integerorder Dirichlet boundary value problems. Based on these new properties and Krasnoselskii fixed point theorem, the existence and multiplicity of positive solutions for BVP (2) were considered. 
In this paper, by using bifurcation techniques, we consider the following boundary value problem of fractional differential equation:

$$
\begin{gathered}
D_{0^{+}}^{\alpha} u(t)+\eta f(t, u(t))=0, \quad t \in(0,1), \\
u(0)=u(1)=0,
\end{gathered}
$$

where $1<\alpha \leq 2, D_{0^{+}}^{\alpha}$ is the standard Riemann-Liouville differentiation, $r>0$ is a given constant, and $f:[0,1] \times$ $\mathbb{R}^{+} \rightarrow \mathbb{R}^{+}$is a given continuous function satisfying some assumptions that will be specified later.

It is remarkable that the method used in references mentioned above was fixed point theorems and the same kind of conditions was used such that the nonlinearity $f(t, u)$ satisfies superlinear or sublinear condition at 0 and $\infty$, which is similar to (A1). To the best of our knowledge, there is no paper studying such fractional differential equations using bifurcation ideas. As we know, the bifurcation technique is widely used in solving BVP of integer-order differential equations (see, e.g., [11-13] and references therein). In [14], by virtue of bifurcation ideas, the authors studied a kind of BVP of differential inclusions. The purpose of present paper is to fill this gap. By using bifurcation techniques and topological degree theory, the existence of positive solutions of BVP (3) is investigated. The main features of present paper are as follows. First, the nonlinearity $f(t, u)$ is asymptotically linear at 0 and $\infty$, not super-linear or sub-linear (see the condition (H1) in Section 2 and example in Section 4). Next, the main method used here is bifurcation techniques and topological degree, not fixed point theorem on cone, which is different from the references.

The paper is organized as described below. At the end of this section, for completeness, we list some results on bifurcation theory from interval and topological degree of completely continuous operators. Section 2 contains background materials and preliminaries. In Section 3, by using bifurcation techniques and topological degree theory, bifurcation results from infinity and trivial solution are established. Then the main results of present paper are given and proved. Finally in Section 4 , an example is worked out to demonstrate the main results.

Lemma 1 (Schmitt and Thompson [15]). Let $V$ be a real reflexive Banach space. Let $G: \mathbb{R} \times V$ to $V$ be completely continuous such that $G(\lambda, 0)=0$, for all $\lambda \in \mathbb{R}$. Let $a, b \in \mathbb{R}(a<b)$ be such that $u=0$ is an isolated solution of the equation

$$
u-G(\lambda, u)=0, \quad u \in V,
$$

for $\lambda=a$ and $\lambda=b$, where $(a, 0),(b, 0)$ are not bifurcation points of (4). Furthermore, assume that

$$
\operatorname{deg}\left(I-G(a, \cdot), B_{r}(0), 0\right) \neq \operatorname{deg}\left(I-G(b, \cdot), B_{r}(0), 0\right),
$$

where $B_{r}(0)$ is an isolating neighborhood of the trivial solution. Let

$$
\begin{aligned}
\mathscr{T}= & \overline{\{(\lambda, u):(\lambda, u) \text { is a solution of }(4) \text { with } u \neq 0\}} \\
& \cup([a, b] \times 0) .
\end{aligned}
$$

Then there exists a connected component $\mathscr{C}$ of $\mathscr{T}$ containing $[a, b] \times 0$ in $\mathbb{R} \times V$, and either

(i) $\mathscr{C}$ is unbounded in $\mathbb{R} \times V$ or

(ii) $\mathscr{C} \cap[(\mathbb{R} \backslash[a, b]) \times 0] \neq \emptyset$.

Lemma 2 (Schmitt [16]). Let $V$ be a real reflexive Banach space. Let $G: \mathbb{R} \times V$ to $V$ be completely continuous, and let $a, b \in \mathbb{R}(a<b)$ be such that the solution of (4) is, a priori, bounded in $V$ for $\lambda=a$ and $\lambda=b$; that is, there exists an $R>0$ such that

$$
G(a, u) \neq u \neq G(b, u)
$$

for all $u$ with $\|u\| \geq R$. Furthermore, assume that

$$
\operatorname{deg}\left(I-G(a, \cdot), B_{R}(0), 0\right) \neq \operatorname{deg}\left(I-G(b, \cdot), B_{R}(0), 0\right),
$$

for $R>0$ sufficiently large. Then there exists a closed connected set $\mathscr{C}$ of solutions of (4) that is unbounded in $[a, b] \times V$, and either

(i) $\mathscr{C}$ is unbounded in $\lambda$ direction or

(ii) there exists an interval $[c, d]$ such that $(a, b) \cap(c, d)=\emptyset$ and $\mathscr{C}$ bifurcates from infinity in $[c, d] \times V$.

Lemma 3 (Guo [17]). Let $\Omega$ be a bounded open set of real Banach space E, and let $A: \bar{\Omega} \rightarrow$ E be completely continuous. If there exists $y_{0} \in E, y_{0} \neq \theta$ such that

$$
x \in \partial \Omega, \quad \tau \geq 0 \Longrightarrow x-A x \neq \tau y_{0},
$$

then

$$
\operatorname{deg}(I-A, \Omega, \theta)=0 .
$$

\section{Background Materials and Preliminaries}

For convenience, we present some necessary definitions and results on fractional calculus theory (see [6]).

Definition 4. The fractional (arbitrary) order integral of the function $h \in L^{1}([a, b])$ of order $\alpha \in \mathbb{R}^{+}$is defined by

$$
I_{a+}^{\alpha} h(t)=\int_{a}^{t} \frac{(t-s)^{\alpha-1}}{\Gamma(\alpha)} h(s) d s,
$$

where $\Gamma$ is the gamma function. When $a=0$, we write $I^{\alpha} h(t)=\left[h * \varphi_{\alpha}\right](t)$, where $\varphi_{\alpha}(t)=t^{\alpha-1} / \Gamma(\alpha)$ for $t>0$, and $\varphi_{\alpha}(t)=0$ for $t \leq 0$, and $\varphi_{\alpha} \rightarrow \delta(t)$ as $\alpha \rightarrow 0$, where $\delta$ is the delta function.

Definition 5. For a function $h$ given on the interval $[a, b]$, the $\alpha$ th Riemann-Liouville fractional-order derivative of $h$ is defined by

$$
\left(D_{a+}^{\alpha} h\right)(t)=\frac{1}{\Gamma(n-\alpha)}\left(\frac{d}{d t}\right)^{n} \int_{a}^{t}(t-s)^{n-\alpha-1} h(s) d s,
$$

where $n=[\alpha]+1$. 
Lemma 6. Let $\alpha>0$; then, the differential equation

$$
D_{0^{+}}^{\alpha} u(t)=0
$$

has solutions $u(t)=c_{1} t^{\alpha-1}+c_{2} t^{\alpha-2}+\cdots+c_{n} t^{\alpha-n}$, for some $c_{i} \in \mathbb{R}, i=0,1,2, \ldots, n$, where $n$ is the smallest integer greater than or equal to $\alpha$.

Notice that $D_{0^{+}}^{\alpha} I^{\alpha} h(t)=h(t)$ for all $h \in C(0,1) \cap L(0,1)$. From Lemma 6 , we deduce the following result.

Lemma 7. Assume that $u \in C(0,1) \cap L^{1}[0,1]$ with a derivative of order $n$ that belongs to $C(0,1) \cap L^{1}[0,1]$. Then

$$
I_{0^{+}}^{\alpha} D_{0^{+}}^{\alpha} u(t)=u(t)+c_{1} t^{\alpha-1}+c_{2} t^{\alpha-2}+\cdots+c_{n} t^{\alpha-n},
$$

for some $c_{i} \in \mathbb{R}, i=0,1,2, \ldots, n$, where $n$ is the smallest integer greater than or equal to $\alpha$.

For more detailed results of fractional calculus, we refer the reader to [6].

Now let us list the following assumption satisfied throughout the paper.

(H1) There exist two positive numbers $\bar{r}, \bar{R}$ with $\bar{r}<\bar{R}$ and functions $a_{0}, a^{0}, b_{\infty}, a^{0} \in C\left(J, \mathbb{R}^{+}\right)$with $a_{0}(t), a^{0}(t)$, $b_{\infty}(t), a^{0}(t) \neq \equiv$ in any subinterval of $[0,1]$ such that

$$
\begin{array}{r}
f(t, u) \subset\left[a_{0}(t)\left(u-\xi_{1}(t, u)\right), a^{0}(t)\left(u+\xi_{2}(t, u)\right)\right], \\
\forall(t, u) \in J \times[0, \bar{r}], \\
f(t, u) \subset\left[b_{\infty}(t)\left(u-\zeta_{1}(t, u)\right), b^{\infty}(t)\left(u+\zeta_{2}(t, u)\right)\right], \\
\forall(t, u) \in J \times[\bar{R},+\infty),
\end{array}
$$

where $J=[0,1], \xi_{i}, \zeta_{i} \in C\left(J \times \mathbb{R}^{+}\right)$with $\xi_{i}\left(t, t^{\alpha-2} u\right)=o\left(t^{\alpha-2} u\right)$ as $u \rightarrow 0$ uniformly with respect to $t \in[0,1](i=1,2)$, and $\zeta_{i}\left(t, t^{\alpha-2} u\right)=o\left(t^{\alpha-2} u\right)$ as $u \rightarrow+\infty$ uniformly with respect to $t \in[0,1](i=1,2)$.

To solve BVP (3), we first consider the following linear boundary problem of fractional differential equation:

$$
\begin{gathered}
D_{0^{+}}^{\alpha} u(t)+g(t)=0, \quad t \in(0,1), \\
u(0)=u(1)=0,
\end{gathered}
$$

where $g \in C[0,1]$. We cite the following two lemmas from references.

Lemma 8 (see [9]). Given $g \in C[0,1]$, then

$$
u(t)=\int_{0}^{1} G(t, s) g(s) d s
$$

is a solution of (16), where

$$
G(t, s)=\frac{1}{\Gamma(\alpha)} \begin{cases}{[t(1-s)]^{\alpha-1}-(t-s)^{\alpha-1},} & 0 \leq s \leq t \leq 1 \\ {[t(1-s)]^{\alpha-1},} & 0 \leq t \leq s \leq 1\end{cases}
$$

Lemma 9 (see [10]). The function $G(t, s)$ defined by (18) has the following properties.

(i) $G(t, s)>0, \forall t, s \in(0,1)$.

(ii) The function $G^{*}(t, s)=: t^{2-\alpha} G(t, s)$ has the following properties:

$$
\begin{aligned}
& \frac{\alpha-1}{\Gamma(\alpha)} t(1-t) s(1-s)^{\alpha-1} \leq G^{*}(t, s) \\
& \leq \frac{1}{\Gamma(\alpha)} s(1-s)^{\alpha-1}, \\
& \forall t, s \in[0,1]
\end{aligned}
$$

The basic space used in this paper is

$$
E=:\{u \in C[0,1]: u(0)=u(1)=0\} .
$$

Obviously, $E$ is a Banach space with norm $\|u\|=\max _{t \in J}|u(t)|$ (for all $u \in E$ ).

Let

$$
Q:=\{v \in E: v(t) \geq(\alpha-1) t(1-t) v(s) \geq 0, \forall s, t \in(0,1)\} \text {. }
$$

It is easy to see that $Q$ is a cone of $E$. Moreover, from (21), we have, for all $v \in Q$,

$$
v(t) \geq(\alpha-1) t(1-t)\|v\|, \quad \forall t \in[0,1] .
$$

For the sake of using bifurcation technique to investigate BVP (3), we study the following fractional boundary value problem with parameter $\lambda$ :

$$
\begin{gathered}
D_{0^{+}}^{\alpha} y(t)+\lambda f(t, y(t))=0, \quad t \in(0,1), \\
y(0)=y(1)=0 .
\end{gathered}
$$

A function $(\lambda, u)$ is said to be a solution of BVP (23) if $(\lambda, u)$ satisfies (23). In addition, if $\lambda>0, u(t)>0$ for $t \epsilon$ $(0,1)$, then $(\lambda, u)$ is said to be a positive solution of BVP $(23)$. Obviously, if $\lambda>0, u \in Q \backslash\{\theta\}$ is a solution of BVP (23), then by (22), we know that $(\lambda, u)$ is a positive solution of BVP (23), where $\theta$ denotes the zero element of Banach space $E$.

Define

$$
\bar{f}(t, u)= \begin{cases}f(t, u), & (t, u) \in J \times \mathbb{R}^{+} \\ f(t, 0), & (t, u) \in J \times(-\infty, 0) .\end{cases}
$$

Then $\bar{f}(t, u) \geq 0$ on $J \times \mathbb{R}$. Let

$$
A_{\lambda} v(t)=: \lambda \int_{0}^{1} G^{*}(t, s) \bar{f}\left(s, s^{\alpha-2} v(s)\right) d s, \quad \forall v \in Q .
$$

By assumption (H1) and using a similar process of the proof of Lemma 4.1 in $[10]$, we know that $A_{\lambda}: C[0,1] \rightarrow Q$ is completely continuous.

From Lemma 8 , if $v \in C[0,1]$ is the the fixed point of operator $A_{\lambda}$, then $y(t)=t^{\alpha-2} v(t)$ is the solution of

$$
\begin{gathered}
D_{0^{+}}^{\alpha} y(t)+\lambda \bar{f}(t, y(t))=0, \quad t \in(0,1), \\
y(0)=y(1)=0 .
\end{gathered}
$$


Let

$$
\Sigma=: \overline{\left\{(\lambda, v) \in \mathbb{R}^{+} \times C[0,1]: v=A_{\lambda} v, v \neq \theta\right\}},
$$

where $\theta$ is the zero element of $C[0,1]$. From Lemma 9 and the definitions of $\bar{f}$ and the cone $Q$, it is easy to see that $\Sigma \subset Q$. Moreover, we have the following conclusion.

Lemma 10. For $\lambda>0$, if $v$ is a nontrivial fixed point of operator $A_{\lambda}$, then $\left(\lambda, t^{\alpha-2} v(t)\right)$ is a positive solution of BVP (23). Furthermore, $(\lambda, y)$ is a positive solution of BVP (23) if and only if $(\lambda, y)$ is a nontrivial solution of BVP (26).

For $a \in C\left(J, \mathbb{R}^{+}\right)$with $a(t) \not \equiv 0$ in any subinterval of $J$, define the linear operator $L_{a}: C(J) \rightarrow C(J)$ by

$$
L_{a} u(t)=\int_{0}^{1} G^{*}(t, s) a(s) s^{\alpha-2} u(s) d s,
$$

where $G^{*}(t, s)$ is defined by Lemma 9 .

From Lemmas 8 and 9 and the well-known Krein-Rutman Theorem, one can obtain the following lemma.

Lemma 11. The operator $L_{a}$ defined by (28) is completely continuous and has a unique characteristic value $\lambda_{1}(a)$, which is positive, real, and simple and the corresponding eigenfunction $\phi(t)$ is of one sign in $(0,1)$; that is, $\phi(t)=\lambda_{1}(a) L_{a} \phi(t)$ for all $t \in J$.

Notice that the operator $L_{a}$ can be regarded as $L_{a}$ : $L^{2}[0,1] \rightarrow L^{2}[0,1]$. This together with Lemma 11 guarantees that $\lambda_{1}(a)$ is also the characteristic value of $L_{a}^{*}$, where $L_{a}^{*}$ is the conjugate operator of $L_{a}$. Let $\varphi^{*}$ denote the nonnegative eigenfunction of $L_{a}^{*}$ corresponding to $\lambda_{1}(a)$. Then we have

$$
\varphi^{*}(t)=\lambda_{1}(a) L_{a}^{*} \varphi^{*}(t), \quad \forall t \in J .
$$

\section{Main Results}

The main results of present paper are the following two theorems.

Theorem 12. Suppose that either

(i) $\eta \in\left[\lambda_{1}\left(a_{0}\right), \lambda_{1}\left(b^{\infty}\right)\right]$ or

(ii) $\eta \in\left[\lambda_{1}\left(b_{\infty}\right), \lambda_{1}\left(a^{0}\right)\right]$.

Then BVP (3) has at least one positive solution.

Theorem 13. Suppose the following.

(H2) There exist $R>0$ and $h \in L[0,1]$ such that

$$
\begin{gathered}
f(t, u) \leq h(t) u, \quad \text { for } t \in[0,1], u \in[0, R], \\
\eta<\frac{\Gamma(\alpha)}{\int_{0}^{1}[s(1-s)]^{\alpha-1} h(s) d s} .
\end{gathered}
$$

In addition, if

$$
\eta>\max \left\{\lambda_{1}\left(a_{0}\right), \lambda_{1}\left(b_{\infty}\right)\right\},
$$

then BVP (3) has at least two positive solutions.
To prove Theorems 12 and 13, we first prove the following lemmas.

Lemma 14. Let $[c, d] \subset \mathbb{R}^{+}$be a compact interval with $\left[\lambda_{1}\left(a^{0}\right), \lambda_{1}\left(a_{0}\right)\right] \cap[c, d]=\emptyset$. Then there exists $\delta_{1} \in(0, \bar{r})$ such that

$$
v \neq A_{\lambda} v, \quad \forall \lambda \in[c, d], \forall v \in E \text { with } 0<\|v\| \leq \delta_{1} .
$$

Proof. If this is false, then there exist $\left\{\left(\mu_{n}, v_{n}\right)\right\} \subset[c, d] \times$ $C[0,1]$ with $\left\|v_{n}\right\| \rightarrow 0+(n \rightarrow+\infty)$ such that $v_{n}=A_{\mu_{n}} v_{n}$. Without loss of generality, assume $\mu_{n} \rightarrow \mu \in[c, d]$ and $\left\|v_{n}\right\|<\bar{r}$ for all $n$. Notice that $v_{n} \in Q$. By Lemma 10 and (21), we have $v_{n}(t)>0$ in $(0,1)$. Set $w_{n}=v_{n} /\left\|v_{n}\right\|$. Then $w_{n}=$ $A_{\mu_{n}} v_{n} /\left\|v_{n}\right\|$. From the definition of $\bar{f}(t, u)$, it is easy to see that $\left\{w_{n}\right\}$ is relatively compact in $C[0,1]$. Taking a subsequence and relabeling if necessary, suppose $w_{n} \rightarrow w$ in $C[0,1]$. Then $\|w\|=1$ and $w \in Q$.

On the other hand, from (H1), we know

$$
\begin{array}{r}
f(t, u) \in\left[a_{0}(t)\left(u-\xi_{1}(t, u)\right), a^{0}(t)\left(u+\xi_{2}(t, u)\right)\right], \\
\forall(t, u) \in J \times[0, \bar{r}] .
\end{array}
$$

Therefore, by virtue of (25), we know

$$
\begin{gathered}
w_{n}(t) \leq \mu_{n} \int_{0}^{1} G^{*}(t, s) a^{0}(s)\left(s^{\alpha-2} w_{n}(s)\right. \\
\left.+\frac{\xi_{2}\left(s, s^{\alpha-2} v_{n}(s)\right)}{\left\|v_{n}\right\|}\right) d s \\
w_{n}(t) \geq \mu_{n} \int_{0}^{1} G^{*}(t, s) a_{0}(s) \\
\quad \times\left(s^{\alpha-2} w_{n}(s)-\frac{\xi_{1}\left(s, s^{\alpha-2} v_{n}(s)\right)}{\left\|v_{n}\right\|}\right) d s .
\end{gathered}
$$

Let $\psi^{*}$ and $\psi_{*}$ be the positive eigenfunctions of $L_{a^{0}}^{*}, L_{a_{0}}^{*}$ corresponding to $\lambda_{1}\left(a^{0}\right)$ and $\lambda_{1}\left(a_{0}\right)$, respectively. Then from (34), it follows that

$$
\begin{aligned}
\left\langle w_{n}, \psi^{*}\right\rangle \leq & \mu_{n}\left\langle L_{a^{0}} w_{n}, \psi^{*}\right\rangle \\
& +\mu_{n} \int_{0}^{1} \psi^{*}(t) \\
& \times \int_{0}^{1} G^{*}(t, s) a_{0}(s) \frac{\xi_{2}\left(s, s^{\alpha-2} v_{n}(s)\right)}{\left\|v_{n}\right\|} d s d t .
\end{aligned}
$$

Letting $n \rightarrow+\infty$ and using condition (H1), we have

$$
\begin{aligned}
\left\langle v, \psi^{*}\right\rangle & \leq \mu\left\langle L_{a^{0}} v, \psi^{*}\right\rangle=\mu\left\langle v, L_{a^{0}}^{*} \psi^{*}\right\rangle \\
& =\mu\left\langle v, \frac{\psi^{*}}{\lambda_{1}\left(a^{0}\right)}\right\rangle,
\end{aligned}
$$

which implies $\mu \geq \lambda_{1}\left(a^{0}\right)$. Similarly, one can deduce from (35) that $\mu \leq \lambda_{1}\left(a_{0}\right)$. 
Consequently, $\lambda_{1}\left(a^{0}\right) \leq \mu \leq \lambda_{1}\left(a_{0}\right)$, which contradicts $\mu \in[c, d]$. Therefore, there exists $\delta_{1} \in(0, \bar{r})$ such that

$v \neq A_{\lambda} v, \quad \forall \lambda \in[c, d], \forall v \in E$ with $0<\|v\| \leq \delta_{1}$.

Lemma 15. For $\mu \in\left(0, \lambda_{1}\left(a^{0}\right)\right)$, there exists $\delta_{1} \in(0, \bar{r})$ such that

$$
\operatorname{deg}\left(I-A_{\mu}, B_{\delta}, 0\right)=1, \quad \forall \delta \in\left(0, \delta_{1}\right] .
$$

Proof. Notice that $[0, \mu] \cap\left[\lambda_{1}\left(a^{0}\right), \lambda_{1}\left(a_{0}\right)\right]=\emptyset$. From Lemma 14 , there exists $\delta_{1} \in(0, \bar{r})$ such that

$$
v \neq A_{\lambda} v, \quad \forall \lambda \in[0, \mu], \forall v \in C[0,1] \text { with } 0<\|v\| \leq \delta_{1},
$$

which means

$$
v \neq \tau A_{\mu} v, \quad \forall \tau \in[0,1], \forall v \in C[0,1] \text { with } 0<\|v\| \leq \delta_{1} .
$$

Therefore, by the homotopy invariance of topological degree, we have

$$
\operatorname{deg}\left(I-A_{\mu}, B_{\delta}, 0\right)=\operatorname{deg}\left(I, B_{\delta}, 0\right)=1, \quad \forall \delta \in\left(0, \delta_{1}\right] .
$$

Lemma 16. For $\lambda>\lambda_{1}\left(a_{0}\right)$, there exists $\delta_{2} \in(0, \bar{r})$ such that

$$
\operatorname{deg}\left(I-A_{\lambda}, B_{\delta}, 0\right)=0, \quad \forall \delta \in\left(0, \delta_{2}\right] .
$$

Proof. First we prove that for $\lambda>\lambda_{1}\left(a_{0}\right)$, there exists $\delta_{2} \epsilon$ $(0, \bar{r})$ such that

$$
v-A_{\lambda} v \neq \tau \varphi_{0}, \quad \forall \tau \geq 0, \forall v \in C[0,1] \text { with } 0<\|v\| \leq \delta_{2},
$$

where $\varphi_{0}$ is the positive eigenfunctions of $L_{a_{0}}$ corresponding to $\lambda_{1}\left(a_{0}\right)$.

If this is false, then there exist $v_{n} \in C[0,1]$ with $\left\|v_{n}\right\| \rightarrow 0$ $(n \rightarrow+\infty)$ and $\tau_{n} \geq 0$ such that

$$
v_{n}-A_{\lambda} v_{n}=\tau_{n} \varphi_{0}
$$

By Lemma 10, we have $v_{n}(t)>0$ in $(0,1)$. From $\lambda>$ $\lambda_{1}\left(a_{0}\right)$, there exists $\sigma>0$ satisfying $\lambda>(1-\sigma) \lambda_{1}\left(a_{0}\right)$. Then condition (H1) guarantees that there exists $\delta \in(0, \bar{r})$ such that $\left|\xi_{1}\left(t, t^{\alpha-2} u\right)\right|<\sigma t^{\alpha-2} u$ for $u \in(0, \delta)$. Noticing $\left\|v_{n}\right\| \rightarrow$ $0(n \rightarrow+\infty)$, there exists $N>0$ such that $\left\|v_{n}\right\|<\delta$ for $n>N$. Consequently, by virtue of (25) and (45), for $n>N$, we know

$$
\begin{aligned}
v_{n}(t)= & A_{\lambda} v_{n}(t)+\tau_{n} \varphi_{0} \\
\geq \lambda \int_{0}^{1} G^{*}(t, s) a_{0}(s) & \quad \times\left(s^{\alpha-2} v_{n}(s)-\xi_{1}\left(s, s^{\alpha-2} v_{n}(s)\right)\right) d s \\
& \geq \lambda(1-\sigma) \int_{0}^{1} G^{*}(t, s) a_{0}(s) s^{\alpha-2} v_{n}(s) d s \\
= & \lambda(1-\sigma) L_{a_{0}} v_{n}(t) .
\end{aligned}
$$

Let $\psi_{*}$ be the positive eigenfunction of $L_{a_{0}}^{*}$ corresponding to $\lambda_{1}\left(a_{0}\right)$. Then

$$
\begin{aligned}
\left\langle v_{n}, \psi_{*}\right\rangle & \geq \lambda(1-\sigma)\left\langle L_{a_{0}} v_{n}, \psi_{*}\right\rangle \\
& =\lambda(1-\sigma)\left\langle v_{n}, L_{a_{0}}^{*} \psi_{*}\right\rangle \\
& =\lambda(1-\sigma)\left\langle v_{n}, \frac{\psi_{*}}{\lambda_{1}\left(a_{0}\right)}\right\rangle .
\end{aligned}
$$

This together with $\left\langle v_{n}, \psi_{*}\right\rangle>0$ guarantees that

$$
\lambda(1-\sigma) \leq \lambda_{1}\left(a_{0}\right)
$$

which is a contradiction. Therefore, (44) holds. By Lemma 3, for each $\lambda>\lambda_{1}\left(a_{0}\right)$, there exists $\delta_{2}>0$ such that

$$
\operatorname{deg}\left(I-A_{\lambda}, B_{\delta}, 0\right)=0, \quad \forall \delta \in\left(0, \delta_{2}\right] .
$$

Theorem 17. $\left[\lambda_{1}\left(a^{0}\right), \lambda_{1}\left(a_{0}\right)\right]$ is a bifurcation interval of positive solutions from the trivial solution for BVP (23); that is, there exists an unbounded component $\mathscr{C}_{0}$ of positive solutions of $B V P(23)$, which meets $\left[\lambda_{1}\left(a^{0}\right), \lambda_{1}\left(a_{0}\right)\right] \times\{0\}$. Moreover, there exists no bifurcation interval of positive solutions from the trivial solution which is disjointed with $\left[\lambda_{1}\left(a^{0}\right), \lambda_{1}\left(a_{0}\right)\right]$.

Proof. By virtue of (27) and Lemma 10, we need only to prove that there exists an unbounded component $\mathscr{C}_{0}$ of $\Sigma$, which meets $\left[\lambda_{1}\left(a^{0}\right), \lambda_{1}\left(a_{0}\right)\right] \times\{0\}$, and there exists no bifurcation interval of $\sum$ from the trivial solution which is disjointed with $\left[\lambda_{1}\left(a^{0}\right), \lambda_{1}\left(a_{0}\right)\right]$.

For fixed $n \in \mathbb{N}$ with $\lambda_{1}\left(a^{0}\right)-(1 / n)>0$, by Lemmas 15 and 16 and their proof, there exists $r>0$ such that all of the conditions of Lemma 1 are satisfied with $G(\lambda, u)=A_{\lambda} u$, $a=\lambda_{1}\left(a^{0}\right)-(1 / n)$, and $b=\lambda_{1}\left(a_{0}\right)+(1 / n)$. This together with Lemma 10 guarantees that there exists a closed connected set $\mathscr{C}_{n}$ of $\Sigma$ containing $\left[\lambda_{1}\left(a^{0}\right)-(1 / n), \lambda_{1}\left(a_{0}\right)+(1 / n)\right] \times 0$ in $\mathbb{R}^{+} \times C[0,1]$. From Lemma 14 , the case (ii) of Lemma 1 cannot occur. Thus, $\mathscr{C}_{n}$ bifurcates from $\left[\lambda_{1}\left(a^{0}\right)-(1 / n), \lambda_{1}\left(a_{0}\right)+1 / n\right] \times$ 0 and is unbounded in $\mathbb{R}^{+} \times C[0,1]$. Moreover, for any closed interval $[c, d] \subset\left[\lambda_{1}\left(a^{0}\right)-1 / n, \lambda_{1}\left(a_{0}\right)+1 / n\right] \backslash\left[\lambda_{1}\left(a^{0}\right), \lambda_{1}\left(a_{0}\right)\right]$, by Lemma 14, there exists $\delta_{1}>0$ such that the set $\{v \in$ $\left.C[0,1]:(\lambda, v) \in \Sigma, 0<\|v\| \leq \delta_{1}, \lambda \in[c, d]\right\}=\emptyset$. Therefore, $\mathscr{C}_{n}$ must be bifurcated from $\left[\lambda_{1}\left(a^{0}\right), \lambda_{1}\left(a_{0}\right)\right] \times\{0\}$, which implies that $\mathscr{C}_{n}$ can be regarded as $\mathscr{C}_{0}$. In addition, using Lemma 14 again, there exists no bifurcation interval of positive solutions from the trivial solution which is disjointed with $\left[\lambda_{1}\left(a^{0}\right), \lambda_{1}\left(a_{0}\right)\right]$.

By a process similar to the above, one can obtain the following conclusions.

Lemma 18. Let $[c, d] \subset \mathbb{R}^{+}$be a compact interval with $\left[\lambda_{1}\left(b^{\infty}\right), \lambda_{1}\left(b_{\infty}\right)\right] \cap[c, d]=\emptyset$. Then there exists $R_{1}>\bar{R}$ such that

$u \neq A_{\lambda} u, \quad \forall \lambda \in[c, d], \forall u \in C[0,1]$ with $\|u\| \geq R_{1}$. 
Lemma 19. For $\mu \in\left(0, \lambda_{1}\left(b^{\infty}\right)\right)$, there exists $R_{1}>\bar{R}$ such that

$$
\operatorname{deg}\left(I-A_{\mu}, B_{R}, 0\right)=1, \quad \forall R \geq R_{1} \text {. }
$$

Lemma 20. For $\lambda>\lambda_{1}\left(b_{\infty}\right)$, there exists $R_{2}>\bar{R}$ such that

$$
\operatorname{deg}\left(I-A_{\lambda}, B_{R}, 0\right)=0, \quad \forall R \geq R_{2} .
$$

Theorem 21. $\left[\lambda_{1}\left(b^{\infty}\right), \lambda_{1}\left(b_{\infty}\right)\right]$ is a bifurcation interval of positive solutions from infinity for $B V P(23)$, and there exists no bifurcation interval of positive solutions from infinity which is disjoint with $\left[\lambda_{1}\left(b^{\infty}\right), \lambda_{1}\left(b_{\infty}\right)\right]$. More precisely, there exists an unbounded component $\mathscr{C}^{\infty}$ of solutions of BVP (23) which meets $\left[\lambda_{1}\left(b^{\infty}\right), \lambda_{1}\left(b_{\infty}\right)\right] \times \infty$ and is unbounded in $\lambda$ direction.

Now we are in position to prove Theorems 12 and 13.

Proof of Theorem 12. Obviously the solution of the form $(\eta, y)$ $(y \neq \theta)$ of $(23)$ is a positive solution of BVP (3). So by Lemma 10, it is sufficient to show that there is a component $\mathscr{C}$ of $\Sigma$ that crosses the hyperplane $\{\eta\} \times C[0,1]$, where $\Sigma \subset$ $\mathbb{R}^{+} \times C[0,1]$ is defined by $(27)$.

Case $i\left(\lambda_{1}\left(a_{0}\right)<\eta<\lambda_{1}\left(b^{\infty}\right)\right)$. By Theorem 17, there exists an unbounded component $\mathscr{C}_{0}$ of positive solutions of BVP (23), which bifurcates from $\left[\lambda_{1}\left(a^{0}\right), \lambda_{1}\left(a_{0}\right)\right] \times\{\theta\}$. Therefore, there exists $\left(\mu_{n}, v_{n}\right) \in \mathscr{C}_{0}$ such that

$$
\mu_{n}+\left\|v_{n}\right\| \longrightarrow+\infty \text { as } n \longrightarrow+\infty \text {. }
$$

If there exists some $n \in \mathbb{N}$ such that $\mu_{n} \geq \eta$, the conclusion follows. Suppose, on the contrary, $\mu_{n}<\eta$ for all $n \in \mathbb{N}$. Since $(0, \theta)$ is the only solution of (23) with $\lambda=0$, by Lemmas 14 and 18, we know $\mathscr{C}_{0} \cap(\{0\} \times C[0,1])=\emptyset$. Therefore, $\mu_{n} \in$ $(0, \eta)$ for all $n \in \mathbb{N}$. Taking a subsequence and relabeling if necessary, suppose $\mu_{n} \rightarrow \mu^{*}$ as $n \rightarrow+\infty$. Then $\mu^{*} \in[0, \eta]$. This together with (53) guarantees that $\left\|v_{n}\right\| \rightarrow+\infty$.

Choose $[c, d]=\left[0, \lambda_{1}\left(b^{\infty}\right)-(1 / m)\right]$ for $m \in \mathbb{N}$. From Lemma 18 , it follows that $\mu^{*}>\lambda_{1}\left(b^{\infty}\right)-(1 / m)$ for each $m \in$ $\mathbb{N}$, which means $\mu^{*} \geq \lambda_{1}\left(b^{\infty}\right)>\eta$. This is a contradiction.

Case ii $\left(\lambda_{1}\left(b_{\infty}\right)<\eta<\lambda_{1}\left(a^{0}\right)\right)$. From Theorem 21 , there exists an unbounded component $\mathscr{C}^{\infty}$ of solutions of (23) which bifurcates from $\left[\lambda_{1}\left(b^{\infty}\right), \lambda_{1}\left(b_{\infty}\right)\right] \times \infty$ and is unbounded in $\lambda$ direction.

If $\mathscr{C}^{\infty} \cap\left(\mathbb{R}^{+} \times\{0\}\right)=\emptyset$, using the fact that $\mathscr{C}^{\infty} \cap(\{0\} \times$ $C[0,1])=\emptyset$ and $\mathscr{C}^{\infty}$ is unbounded in $\lambda$ direction, we know that $\mathscr{C}^{\infty}$ must cross the hyperplane $\{\eta\} \times C[0,1]$.

If $\mathscr{C}^{\infty} \cap\left(\mathbb{R}^{+} \times\{0\}\right) \neq \emptyset$, by $\mathscr{C}^{\infty} \cap(\{0\} \times C[0,1])=\emptyset$ and Theorem 17, we know $\mathscr{C}^{\infty} \cap\left(\mathbb{R}^{+} \times\{0\}\right) \in\left[\lambda_{1}\left(a^{0}\right), \lambda_{1}\left(a_{0}\right)\right] \times$ $\{0\}$. Therefore, $\mathscr{C}^{\infty}$ joins $\left[\lambda_{1}\left(a^{0}\right), \lambda_{1}\left(a_{0}\right)\right] \times\{0\}$ to $\left[\lambda_{1}\left(b^{\infty}\right)\right.$, $\left.\lambda_{1}\left(b_{\infty}\right)\right] \times \infty$. This together with $\lambda_{1}\left(b_{\infty}\right)<\eta<\lambda_{1}\left(a^{0}\right)$ guarantees that $\mathscr{C}^{\infty}$ crosses the hyperplane $\{\eta\} \times C[0,1]$.

Proof of Theorem 13. First we show that there exists $\varepsilon>0$ such that

$$
\Sigma \cap\left([0, \eta+\varepsilon] \times \partial B_{R}\right)=\emptyset
$$

where $B_{R}=\{v \in C[0,1]:\|v\|<R\}, \Sigma \subset \mathbb{R}^{+} \times C[0,1]$ is defined by (27).
In fact, from assumption ( $\mathrm{H} 2)$, it follows that there exists $\varepsilon>0$ such that

$$
\frac{\eta+\varepsilon}{\Gamma(\alpha)} \int_{0}^{1}[s(1-s)]^{\alpha-1} h(s) d s<1 .
$$

If there is a solution $(\lambda, v)$ of $v=A_{\lambda} v$ such that $0 \leq \lambda \leq \eta+\varepsilon$ and $\|v\|=R$, then

$$
0 \leq v(t) \leq\|v\|=R \quad \text { for } t \in[0,1] .
$$

By virtue of (25) and Lemma 9, we have

$$
\begin{aligned}
R & =\|v\|=\max _{t \in J} \lambda \int_{0}^{1} G^{*}(t, s) \bar{f}\left(s, s^{\alpha-2} v(s)\right) d s \\
& \leq(\eta+\varepsilon) R \max _{t \in J} \int_{0}^{1} G^{*}(t, s) s^{\alpha-2} h(s) d s \\
& \leq \frac{(\eta+\varepsilon) R}{\Gamma(\alpha)} \int_{0}^{1}[s(1-s)]^{\alpha-1} h(s) d s<R,
\end{aligned}
$$

which is a contradiction. Thus, $\Sigma \cap\left([0, \eta+\varepsilon] \times \partial B_{R}\right)=\emptyset$.

Next, from Theorem 17, there exist unbounded components $\mathscr{C}_{0}$ of solutions of $(23)$, which meet $\left[\lambda_{1}\left(a^{0}\right), \lambda_{1}\left(a_{0}\right)\right] \times$ $\{0\}$. By $(54)$, we know $\mathscr{C}_{0} \cap\left([0, \eta+\varepsilon] \times \partial B_{R}\right)=\emptyset$. This together with the fact that $\mathscr{C}_{0}$ is unbounded, $\lambda_{1}\left(a_{0}\right)<\eta$, and $\mathscr{C}_{0} \cap$ $(\{0\} \times C[0,1])=\emptyset$ guarantees that $\mathscr{C}_{0}$ crosses the hyperplane $\{\eta\} \times C[0,1]$. Then $\operatorname{BVP}(23)$ has a positive solution $v_{1}$ with $\left(\eta, v_{1}\right) \in \mathscr{C}_{0}$ and $\left\|v_{1}\right\|<R$. By Lemma $10, t^{\alpha-2} v_{1}(t)$ is a positive solution of BVP (3).

Similarly, by Theorem 21 and (54), BVP (3) has a positive solution $t^{\alpha-2} v_{2}(t)$ with $\left(\eta, v_{2}\right) \in \mathscr{C}^{\infty}$ and $\left\|v_{2}\right\|>R$. The conclusion follows.

Immediately, from the proof of Theorem 13, we have the following corollary.

Corollary 22. Suppose that assumption (H2) holds. In addition, suppose that one of the following two conditions holds:

(i) $\lambda_{1}\left(a_{0}\right)<\eta$;

(ii) $\lambda_{1}\left(b_{\infty}\right)<\eta$.

Then BVP (3) has at least one positive solution.

Remark 23. Corollary 22 is different from Theorem 12 though their results are similar.

\section{An Example}

Let $\rho$ be the unique characteristic value of $L_{1}$ corresponding to positive eigenfunctions with $a(t) \equiv t$ in (28). From Lemma 11, it follows that $\rho$ exists. Now we are ready to give the following example.

Example 1. Consider the following boundary value problem of fractional differential inclusions:

$$
\begin{gathered}
D_{0^{+}}^{1.5} u(t)+\eta f(t, u(t))=0, \quad t \in(0,1), \\
u(0)=u(1)=0,
\end{gathered}
$$


where

$$
\begin{gathered}
f(t, u)=\rho t u\left[h(u)+\sin \frac{1}{u}+t \sin (t u)\right], \\
h(u)= \begin{cases}\frac{3}{2}, & u \in\left(0, \frac{1}{2}\right] \\
1+u, & u \in\left[\frac{1}{2}, 3\right) \\
4, & u \in[3,+\infty) .\end{cases}
\end{gathered}
$$

Then BVP (58) has at least one positive solution as $\eta \in$ $[1 / 3,2 / 5]$.

Proof. BVP (58) can be regarded as the form (3). Let $f(t, u)=$ 0 for $u=0$; then, $f(t, u)$ is continuous.

From (59), choose $\bar{r}=1 / 2, \bar{R}=3, a_{0}(t)=\rho t / 2, a^{0}(t)=$ $(5 / 2) \rho t, b_{\infty}(t)=3 \rho t, b^{\infty}(t)=5 \rho t, \xi_{1}(t, u)=-2 t u \sin (t u)$, $\xi_{2}(t, u)=(2 / 5) t u \sin (t u), \zeta_{1}(t, u)=-(1 / 3) u \sin (1 / u)$, $\zeta_{2}(t, u)=(1 / 5) u \sin (1 / u)$

It is easy to see $\xi_{i}\left(t, t^{\alpha-2} u\right)=o\left(t^{\alpha-2} u\right)$ as $u \rightarrow 0$ and $\zeta_{i}\left(t, t^{\alpha-2} u\right)=o\left(t^{\alpha-2} u\right)$ as $u \rightarrow+\infty$ both uniformly with respect to $t \in[0,1],(i=1,2)$.

Therefore, $(\mathrm{H} 1)$ is satisfied.

By the definition of $\rho$, it is easy to see $\lambda_{1}\left(a^{0}\right)=2 / 5$, $\lambda_{1}\left(b_{\infty}\right)=1 / 3$.

As a result, by Theorem 12, BVP (58) has at least one positive solution as $\eta \in[1 / 3,2 / 5]$.

\section{Acknowledgments}

The author wishes to thank the anonymous referees for their valuable suggestions. Research is supported by the NNSF of China (11171192), the Graduate Educational Innovation Foundation of Shandong Province (SDYY1005), and the Natural Science Foundation of Shandong Province (ZR2013AM005).

\section{References}

[1] B. Ahmad and J. J. Nieto, "Existence of solutions for nonlocal boundary value problems of higher-order nonlinear fractional differential equations," Abstract and Applied Analysis, vol. 2009, Article ID 494720, 9 pages, 2009.

[2] K. Diethelm, The Analysis of Fractional Differential Equations: An Application-Oriented Exposition Using Differential Operators of Caputo Type, vol. 2004, Springer, Berlin, Germany, 2010.

[3] A. A. Kilbas and J. J. Trujillo, "Differential equations of fractional order: methods, results and problems-I," Applicable Analysis, vol. 78, no. 1-2, pp. 153-192, 2001.

[4] A. A. Kilbas and J. J. Trujillo, "Differential equations of fractional order: methods, results and problems-II," Applicable Analysis, vol. 81, no. 2, pp. 435-493, 2002.

[5] I. Podlubny, Fractional Differential Equations, vol. 198 of Mathematics in Science and Engineering, Academic Press, San Diego, Calif, USA, 1999.

[6] S. G. Samko, A. A. Kilbas, and O. I. Marichev, Fractional Integral and Derivatives (Theory and Applications), Gordon and Breach, Yverdon, Switzerland, 1993.

[7] Z. Wei, Q. Li, and J. Che, "Initial value problems for fractional differential equations involving Riemann-Liouville sequential fractional derivative," Journal of Mathematical Analysis and Applications, vol. 367, no. 1, pp. 260-272, 2010.

[8] C. Tian and Y. Liu, "Multiple positive solutions for a class of fractional singular boundary value problems," Georgian Academy of Sciences, vol. 56, pp. 115-131, 2012.

[9] Z. Bai and H. Lü, "Positive solutions for boundary value problem of nonlinear fractional differential equation," Journal of Mathematical Analysis and Applications, vol. 311, no. 2, pp. 495505, 2005.

[10] D. Jiang and C. Yuan, "The positive properties of the Green function for Dirichlet-type boundary value problems of nonlinear fractional differential equations and its application," Nonlinear Analysis. Theory, Methods \& Applications, vol. 72, no. 2, pp. 710-719, 2010.

[11] Y. Liu and D. O'Regan, "Bifurcation techniques for Lidstone boundary value problems," Nonlinear Analysis. Theory, Methods \& Applications, vol. 68, no. 9, pp. 2801-2812, 2008.

[12] R. Ma and J. Xu, "Bifurcation from interval and positive solutions of a nonlinear fourth-order boundary value problem," Nonlinear Analysis. Theory, Methods \& Applications, vol. 72, no. 1, pp. 113-122, 2010.

[13] J. Xu and R. Ma, "Bifurcation from interval and positive solutions for second order periodic boundary value problems," Applied Mathematics and Computation, vol. 216, no. 8, pp. 24632471, 2010.

[14] Y. Liu and H. Yu, "Bifurcation of positive solutions for a class of boundary value problems of fractional differential inclusions," Abstract and Applied Analysis, vol. 2013, Article ID 942831, 8 pages, 2013.

[15] K. Schmitt and R. C. Thompson, Nonlinear Analysis and Differential Equations: An Introduction, University of Utah Lecture Note, Salt Lake City, Utah, USA, 2004.

[16] K. Schmitt, "Positive solutions of semilinear elliptic boundary value problems," in Topological Methods in Differential Equations and Inclusions, vol. 472, pp. 447-500, Kluwer Academic Publishers, Dordrecht, The Netherlands, 1995.

[17] D. Guo, Nonlinear Functional Analysis, Shandong Science and Technology Press, Jinan, China, 2001, (Chinese). 


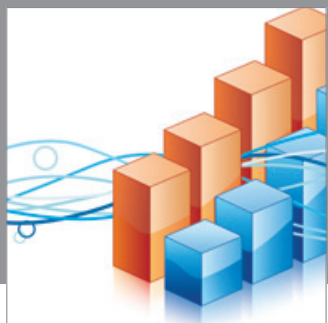

Advances in

Operations Research

mansans

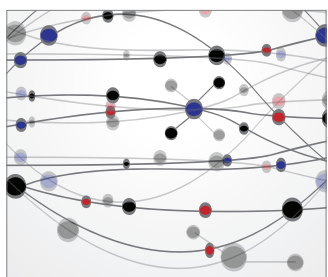

The Scientific World Journal
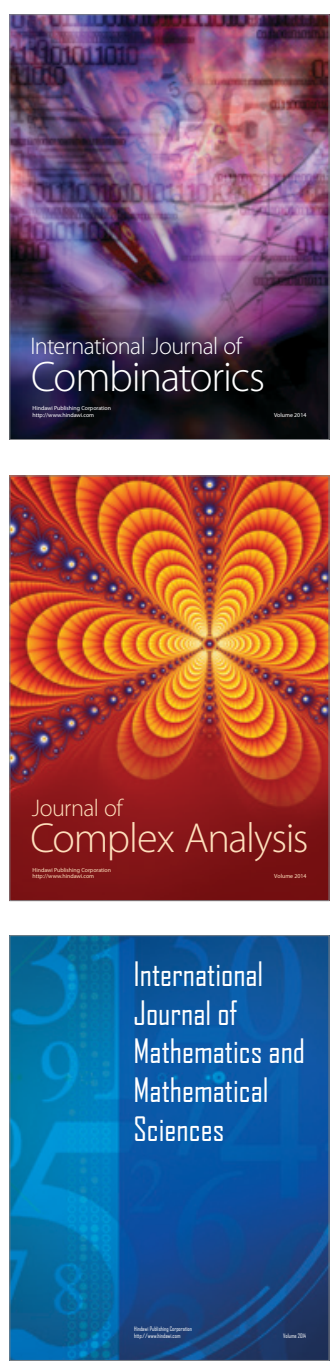
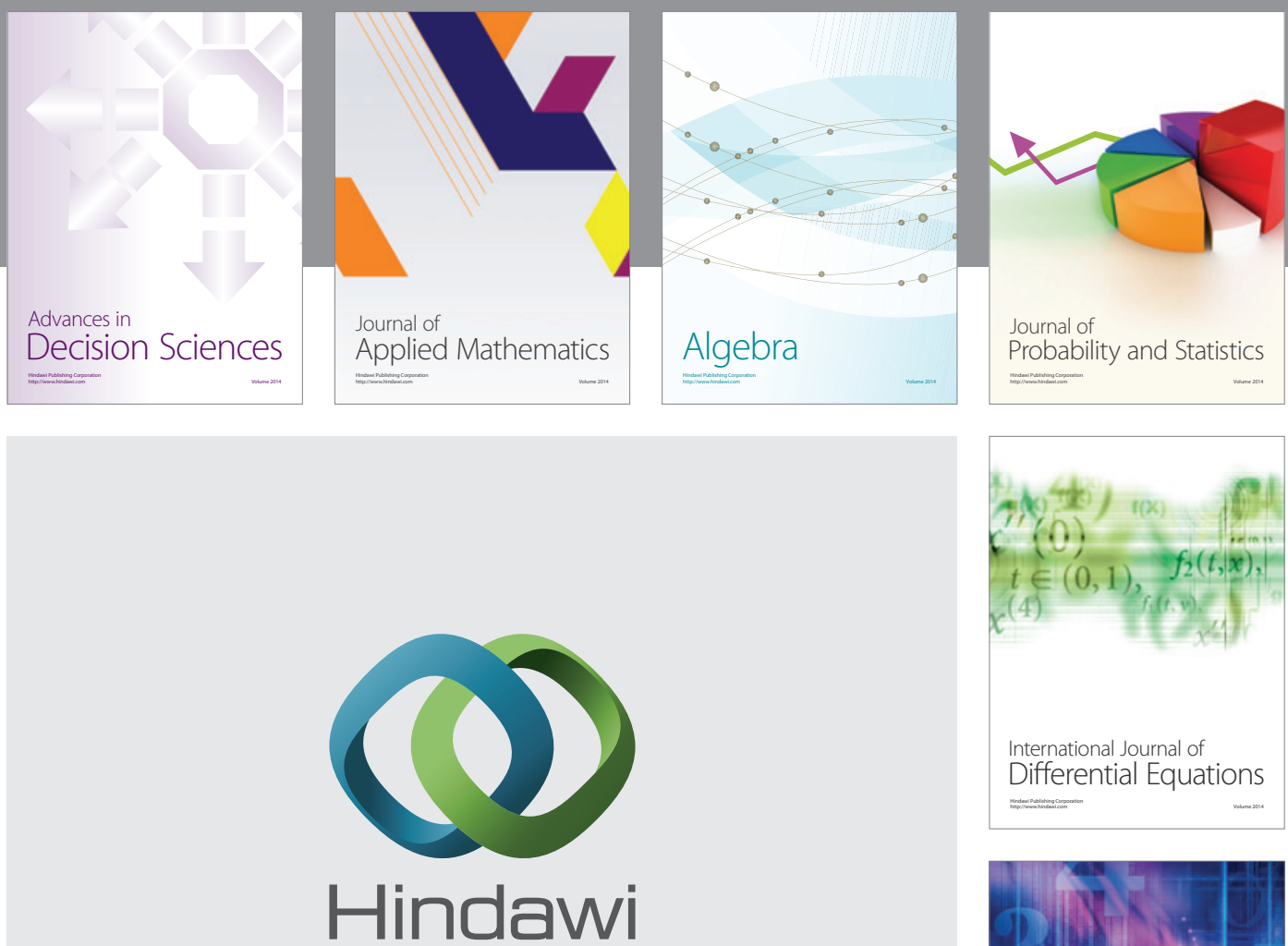

Submit your manuscripts at http://www.hindawi.com
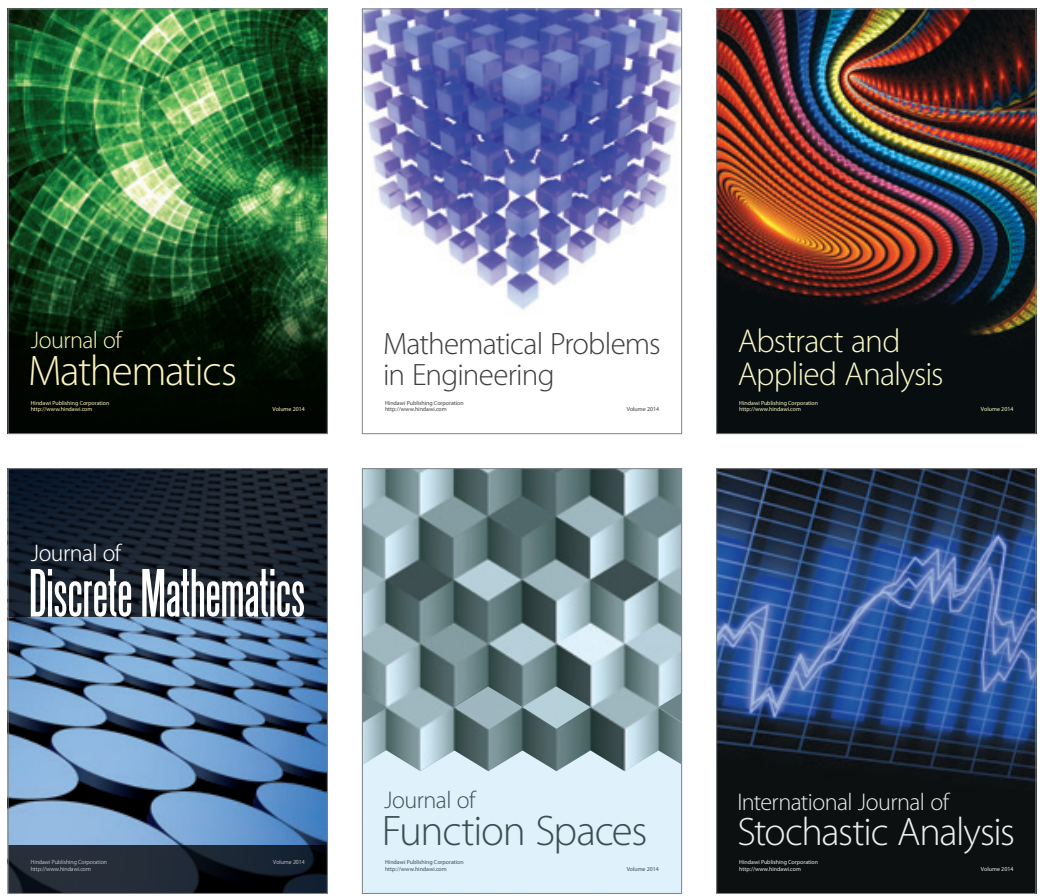

Journal of

Function Spaces

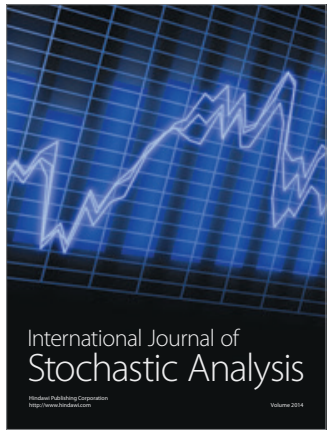

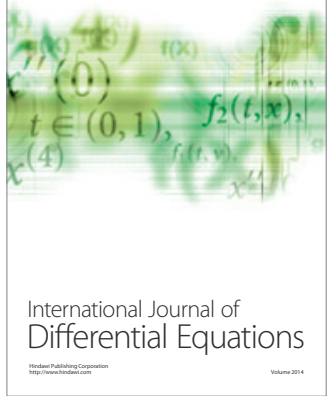
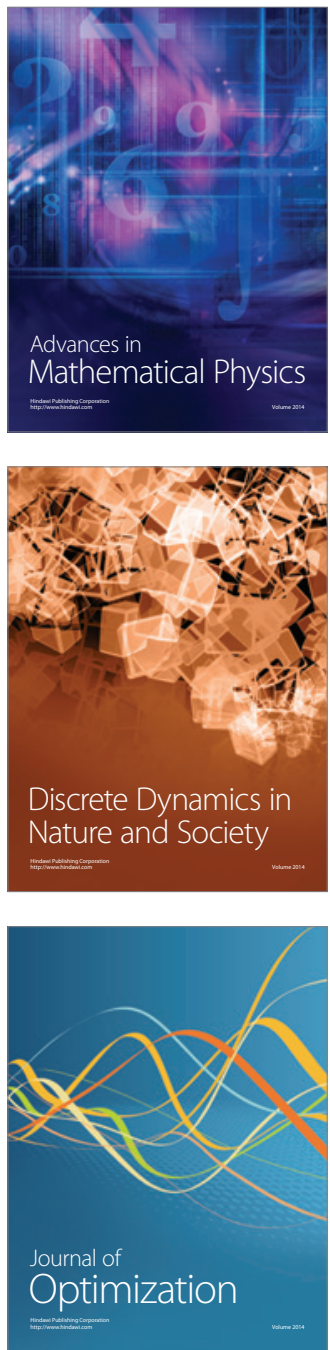